\title{
Registration of \\ 4D Time-Series of Cardiac Images with Multichannel Diffeomorphic Demons
}

\author{
Jean-Marc Peyrat ${ }^{1}$, Hervé Delingette ${ }^{1}$, Maxime Sermesant ${ }^{1,2}$, Xavier Pennec ${ }^{1}$, \\ Chenyang $\mathrm{Xu}^{3}$, and Nicholas Ayache ${ }^{1}$ \\ ${ }^{1}$ INRIA - Asclepios Research Project, Sophia Antipolis, France \\ herve.delingette@sophia.inria.fr \\ ${ }^{2}$ King's College London, Cardiac MR Research Group, Guy's Hospital, London, UK \\ 3 Siemens Corporate Research, Princeton, New Jersey, USA
}

\begin{abstract}
In this paper, we propose a generic framework for intersubject non-linear registration of $4 \mathrm{D}$ time-series images. In this framework, spatio-temporal registration is defined by mapping trajectories of physical points as opposed to spatial registration that solely aims at mapping homologous points. First, we determine the trajectories we want to register in each sequence using a motion tracking algorithm based on the Diffeomorphic Demons algorithm. Then, we perform simultaneously pairwise registrations of corresponding time-points with the constraint to map the same physical points over time. We show this trajectory registration can be formulated as a multichannel registration of $3 \mathrm{D}$ images. We solve it using the Diffeomorphic Demons algorithm extended to vector-valued 3D images. This framework is applied to the inter-subject non-linear registration of $4 \mathrm{D}$ cardiac $\mathrm{CT}$ sequences.
\end{abstract}

\section{Introduction}

In the last decade, the improvement of medical imaging technologies extended $3 \mathrm{D}$ volume acquisitions to $4 \mathrm{D}$ time-series volume data such as Cine MRI, Tagged MRI, 4D CT or 4D ultrasound. They give access to additional information important for studying the motion of organs (such as cardiac and pulmonary) or for real-time control during image-guided surgical procedures. Since the temporal dimension cannot be considered as an additional spatial dimension, the extension of $3 \mathrm{D}$ image processing tools to $4 \mathrm{D}$ data is not obvious. Thus, the development of specific algorithms for spatio-temporal data is necessary such as segmentation [1], tracking [23], temporal alignment 44] and spatio-temporal alignment 64. This last task has been widely studied for multi-view camera registration but mainly limited to linear transformations [6. Perperidis et al. [4] proposed a spatio-temporal non-linear alignment algorithm where they determine a single average free-form deformation between two cardiac sequences for building probabilistic atlas of shape and motion. In such case, due to the cardiac motion, the mapping between voxels at corresponding times has no real physical meaning. In order to correctly handle cardiac motion, a time-dependent 
non-linear transformation is needed to generate an exact mapping between homologous physical points. Furthermore, to ensure a mapping of the same physical points over the entire sequence, the transformation has to be temporally consistent with the motion occurring in both sequences and needs to, hence, map homologous trajectories.

In this paper, we propose a registration framework based on trajectory constraints: the trajectories estimated from the motion tracking in each sequence constrain the mapping to the same physical point over the entire sequence. We start by introducing concepts about spatio-temporal pairwise registration. Under trajectory constraints, spatio-temporal registration can be formulated as a multichannel spatial registration. We solve it by proposing a novel multichannel version of the Diffeomorphic Demons algorithm. Finally, we apply this framework to the inter-subject registration of two 4D cardiac CT sequences.

\section{Trajectory Constrained 4D Registration}

\subsection{Spatio-temporal Registration}

Let $\Omega \times \tau \subset \mathbb{R}^{3} \times \mathbb{R}$ be the acquisition space-time of the reference time-series of images $I$, and $\Omega^{\prime} \times \tau^{\prime} \subset \mathbb{R}^{3} \times \mathbb{R}$ the acquisition space-time in the target time-series of images $I^{\prime}$.

$$
\begin{aligned}
I: \Omega \times \tau & \longrightarrow \mathbb{R} & I^{\prime}: \Omega^{\prime} \times \tau^{\prime} & \longrightarrow \mathbb{R} \\
(x, t) & \longmapsto I(x, t) & \left(x^{\prime}, t^{\prime}\right) & \longmapsto I^{\prime}\left(x^{\prime}, t^{\prime}\right)
\end{aligned}
$$

When registering the target sequence $I^{\prime}$ to the reference sequence $I$, the spatiotemporal transformation $S$ that maps a spatio-temporal position $(x, t)$ of $I$ to the corresponding spatio-temporal position $\left(x^{\prime}, t^{\prime}\right)$ of $I^{\prime}$ must be found:

$$
\begin{aligned}
S: \Omega \times \tau & \longrightarrow \Omega^{\prime} \times \tau^{\prime} \\
(x, t) & \longmapsto S(x, t)=\left(x^{\prime}, t^{\prime}\right)
\end{aligned}
$$

The spatio-temporal transformation $S$ is a combination of a spatial transformation $x^{\prime}=S_{\text {space }}(x, t)$ and a temporal transformation $t^{\prime}=S_{\text {time }}(x, t)$. We make the reasonable assumption that the temporal transformation $S_{\text {time }}$ is only time dependent : $t^{\prime}=S_{\text {time }}(t)$. This transformation is determined by the temporal registration of some quantities that may not be intensity-based. For instance, in cardiac imaging one can use the ECG or the volume curves of the left ventricle as proposed by Perperidis et al. 4. This temporal transformation $S_{\text {time }}$ can be computed after the spatial transformation $S_{\text {space }}$ as long as the acquisition time intervals $\tau$ and $\tau^{\prime}$ exactly overlap. This can be performed by a global affine temporal transformation that is often implicitly performed in $4 \mathrm{D}$ cardiac sequences acquired from the end of diastole (ED) of a cardiac cycle to the end of diastole of the next cycle. Without loss of generality, we can assume that both sequences are already temporally aligned to perform the spatial registration.

The temporal discretization of the $4 \mathrm{D}$ registration is illustrated in Fig. 11 where the spatial transformations $S_{j}$ maps the reference volume $I_{j}$ to the target volume 

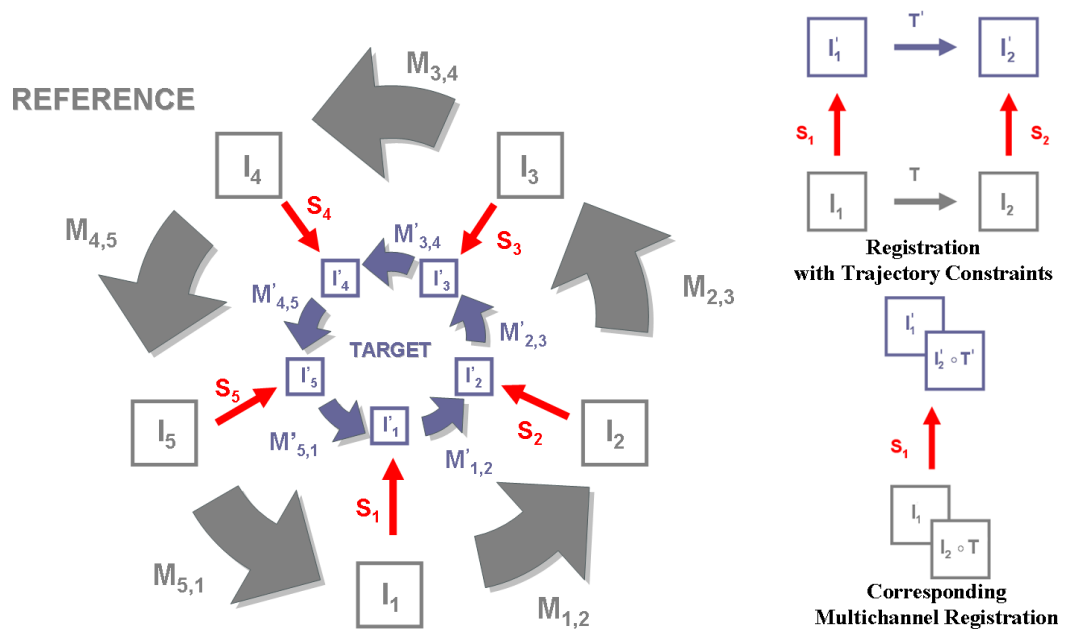

Fig. 1. [Left] Discretization of the 4 D registration with the shape transformations $S_{j}$ between the sequences and the motion transformations $M_{j, j+1}$ and $M_{j, j+1}^{\prime}$ between consecutive frames of a sequence at a given time $t_{j}$; [Right] The simultaneous registration of two pairs of images through the transformations $S_{1}$ and $S_{2}$ knowing the transformations $T$ and $T^{\prime}$ can be formulated as a multichannel registration where $S_{2}$ is computed from $S_{1}$ to satisfy the trajectory constraint: $S_{2}=T^{\prime} \circ S_{1} \circ T^{-1}$

$I_{j}^{\prime}$ at time $t_{j}$ knowing the trajectories of points given by the motion tracking $M_{j, j+1}$ and $M_{j, j+1}^{\prime}$ between the times $t_{j}$ and $t_{j+1}$ respectively in the reference and target sequences. In the remainder, we formulate the $4 \mathrm{D}$ image registration as the minimization of a functional that includes trajectory constraints.

\subsection{Including Trajectory Constraints}

To be physically meaningful when determining the time-dependent spatial trans-

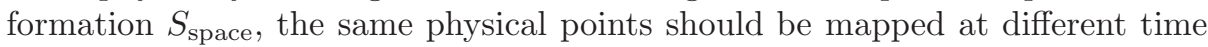
points in both sequences. Using the notations introduced in the previous section, this is equivalent to stating that if a point $x$ in image $I_{j}$ maps a point $x^{\prime}$ in $I_{j}^{\prime}$ by shape transformation $S_{j}$, then the remaining shape transformations $S_{j+1}$ should map the displaced point $M_{j, j+1}(x)$ to the displaced point $M_{j, j+1}^{\prime}\left(x^{\prime}\right)$. This translates into a set of constraints that link the shape transformations $S_{j}$ and $S_{k}$ to the motion transformations $M_{j, k}$ from $I_{j}$ to $I_{k}$ and $M_{j, k}^{\prime}$ from $I_{j}^{\prime}$ to $I_{k}^{\prime}$ :

$$
S_{k} \circ M_{j, k}=M_{j, k}^{\prime} \circ S_{j}
$$

When determining the shape transformation $S_{j}$, the classical approach is to minimize the image similarity measure between image $I_{j}$ and $I_{j}^{\prime}$ :

$$
S_{j}^{3 D}=\underset{S}{\operatorname{argmin}}\left(\int_{\nu \in \Gamma_{j}} \operatorname{Sim}\left(I_{j}(\nu), I_{j}^{\prime} \circ S(\nu)\right) d \nu\right)
$$


We call this shape transformation $S_{j}^{3 D}$ the solution to the $3 \mathrm{D}$ registration problem that only involves one pair of images $\left(I_{j}, I_{j}^{\prime}\right)$. To take into account the fact that $S_{j}$ should map homologous point trajectories and not only homologous points, the trajectory constraints of Equation 1 should be satisfied. Those constraints may be reformulated as $S_{k}=M_{j, k}^{\prime} \circ S_{j} \circ M_{j, k}^{-1}$ which may be interpreted as follows : to satisfy the trajectory constraints, the transformation $M_{j, k}^{\prime} \circ S_{j} \circ M_{j, k}^{-1}$ should map image $I_{k}$ into image $I_{k}^{\prime}$. Therefore, we minimize the following functional to determine the $4 \mathrm{D}$ shape transformation $S_{j}^{4 D}$ :

$$
S_{j}^{4 D}=\underset{S}{\operatorname{argmin}}\left(\sum_{k \neq j} \int_{\nu \in \Gamma_{k}} \operatorname{Sim}\left(I_{k}(\nu), I_{k}^{\prime} \circ M_{j, k}^{\prime} \circ S \circ M_{j, k}^{-1}(\nu)\right) d \nu\right)
$$

Making the following change of variable $\nu=M_{j, k}(\omega)$, the $4 \mathrm{D}$ shape registration can be formulated as the minimization of similarity criterion between several pairs of images (cf. Fig. 1):

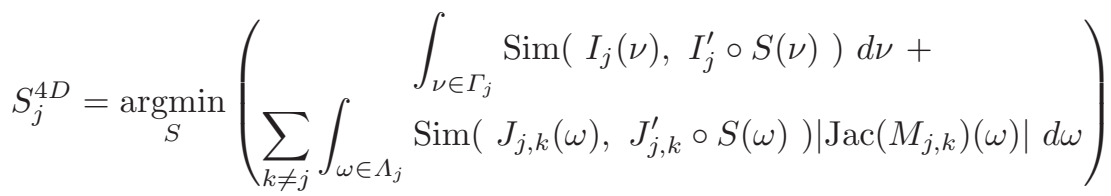

where $J_{j, k}=I_{j} \circ M_{j, k}$ and $J_{j, k}^{\prime}=I_{j}^{\prime} \circ M_{j, k}^{\prime}$ are respectively the images at frame $k$ transformed into the geometry of the image at frame $j$ in the reference and target sequences, $\Lambda_{j} \in \Gamma_{j}$ is part of image $I_{j}$ and $\operatorname{Jac}\left(M_{j, k}\right)$ is the Jacobian of transformation $M_{j, k}$. In other words, the shape transformation $S_{j}^{4 D}$ must optimize the sum of similarity criteria between the pair of images $\left(I_{j}, I_{j}^{\prime}\right)$ and all pairs of images $\left(J_{j, k}, J_{j, k}^{\prime}\right)$. Note also that $\operatorname{Jac}\left(M_{j, k}\right)$ appears to take into account volume change of voxels when transforming $I_{k}$ (resp. $\left.I_{k}^{\prime}\right)$ into $J_{j, k}$ (resp. $J_{j, k}^{\prime}$ ) and acts as a voxel-wise confidence map in each similarity criterion.

Therefore, we have shown that including trajectory constraints in the estimation of the shape registrations $S_{j}$ transforms a $4 \mathrm{D}$ registration problem into a $3 \mathrm{D}$ multichannel registration problem associated with pair of images $\left(I_{k}, I_{k}^{\prime}\right)$ transformed in the space of images $I_{j}$ and $I_{j}^{\prime}$. A novel multichannel registration algorithm based on the Diffeomorphic Demons is presented in the next section.

\section{Multichannel Diffeomorphic Demons}

The Demons algorithm [7 based on optical flow registers the target 3D volume $V^{\prime}$ to the reference $3 \mathrm{D}$ volume $V$ determining an update vector field $\mathbf{u}$ of the current transformation $S$ that minimizes the energy:

$$
E(\mathbf{u})=\frac{1}{2|\Omega|} \int_{\omega \in \Omega}\left\|\left[\begin{array}{c}
V(\omega)-V^{\prime} \circ S(\omega) \\
0
\end{array}\right]+\left[\begin{array}{c}
\mathbf{J}^{T}(\omega) \\
\frac{\sigma(\omega)}{\sigma_{x}} \mathrm{Id}
\end{array}\right] \cdot \mathbf{u}(\omega)\right\|^{2} \alpha(\omega) d \omega
$$


where $\Omega$ is the overlap between $V$ and $V^{\prime} \circ S, \omega$ is the voxel position, $\mathbf{J}$ is the gradient vector in the reference image $V, \sigma_{x}$ is the spatial uncertainty on the correspondences, and $\alpha$ the voxel-wise confidence map. A closed form solution of this minimization is given by the update vector field $\mathbf{u}=-\frac{V-V^{\prime}}{\mathbf{J}^{T} \cdot \mathbf{J}+\frac{\sigma^{2}}{\sigma_{x}^{2}}} \mathbf{J}$. The Diffeomorphic Demons algorithm [8] constrains the deformation field to be diffeomorphic by projecting the update vector field to the one-parameter subgroup of diffeomorphisms with stationary speed vector fields.

An extension of the Demons algorithm to multichannel registration was proposed for DT-MRI registration based on transformation invariant tensor characteristics 9]. The update vector field is computed independently for each channel and then averaged. With this approach, the averaging approximates the coupling between the channels. Yeo et al. 10, preserved their coupling by extending the Demons algorithm to vector-valued images. They also included the finite-strain differential to take into account the reorientation of diffusion tensors. In our case, we work on data that do not need to be reoriented, but we include a voxelwise confidence map for each channel. Thus, we can formulate the multichannel Demons functional as follows:

$$
E(\mathbf{u})=\sum_{j=1}^{N}\left(\frac{1}{2\left|\Omega_{j}\right|} \int_{\omega \in \Omega_{j}}\left\|\left[\begin{array}{c}
V_{j}(\omega)-V_{j}^{\prime} \circ S(\omega) \\
0
\end{array}\right]+\left[\begin{array}{c}
\mathbf{J}_{j}^{T}(\omega) \\
\frac{\sigma_{j}(\omega)}{\sigma_{x}} \mathrm{Id}
\end{array}\right] \cdot \mathbf{u}(\omega)\right\|^{2} \alpha_{j}(\omega) d \omega\right)
$$

where $N$ is the number of channels, $\Omega_{j}$ is the overlap between $V_{j}$ and $V_{j}^{\prime}, \mathbf{J}_{j}$ is the gradient vector in the reference image $V_{j}$, and $\alpha_{j}>0$ the voxel-wise confidence map for channel $j$. Its minimization gives the following equation to solve at each voxel $\sum_{j=1}^{N} \alpha_{j}\left(\mathbf{J}_{j} \cdot \mathbf{J}_{j}^{T}+\frac{\sigma_{j}^{2}}{\sigma_{x}^{2}} \mathrm{Id}\right) \mathbf{u}=-\sum_{j=1}^{N} \alpha_{j}\left(V_{j}-V_{j}^{\prime} \circ S\right) \mathbf{J}_{j}$. Considering the eigen decomposition $\sum_{i=1}^{3} \lambda_{i}^{2} \mathbf{e}_{i} \cdot \mathbf{e}_{i}^{T}$ of the $3 \times 3$ symmetric positive $\operatorname{matrix} D=\sum_{j=1}^{N} \alpha_{j} \mathbf{J}_{j} . \mathbf{J}_{j}^{T}$, the update vector field becomes $\mathbf{u}=\sum_{i=1}^{3} \frac{P_{i}}{\lambda_{i}^{2}+\frac{\sigma^{2}}{\sigma_{x}^{2}}} \mathbf{e}_{i}$, where $P_{i}=\left(\sum_{j=1}^{N} \alpha_{j}\left(V_{j}-V_{j}^{\prime} \circ S\right) \mathbf{J}_{j}^{T}\right) \cdot \mathbf{e}_{i}$ and $\sigma^{2}=\sum_{j=1}^{N} \alpha_{j} \sigma_{j}^{2}$. The coupling between channels relies on the eigen decomposition of the sum $D$ of the dyadic tensors $\alpha_{j} \mathbf{J}_{j} . \mathbf{J}_{j}^{T}$.

\section{Experiments on Cardiac CT Sequences}

We apply this framework to a pair of $4 \mathrm{D}$ cardiac $\mathrm{CT}$ sequences acquired at a spatial resolution of $0.825 \times 0.825 \times 1.00 \mathrm{~mm}^{3}$ with $256 \times 256 \times 231$ voxels. The temporal acquisition is synchronized to the ECG from ED over a cardiac cycle with 20 frames. Given this acquisition process, the affine temporal registration between both sequences is already performed. We performed an anisotropic smoothing and downsampled the data to $1.65 \times 1.65 \times 3.0 \mathrm{~mm}^{3}$ with $128 \times 128 \times 64$ voxels while preserving the temporal resolution. The Diffeomorphic Demons [8] is performed on pairs of successive frames to determine each motion transformations $M_{j, k}$ and $M_{j, k}^{\prime}$ in the reference and target sequences. The transformation 

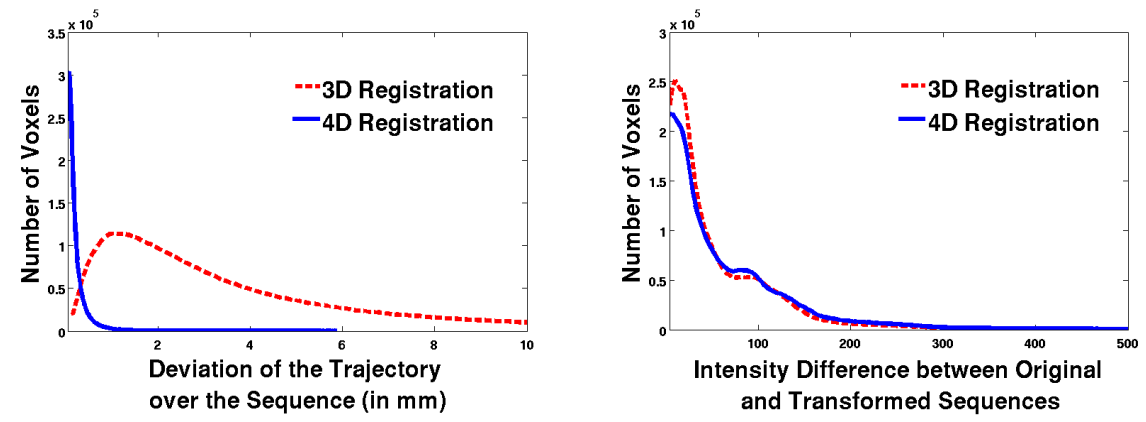

Fig. 2. Cumulated histograms over the entire sequence for the 3D (dashed red curve) and 4D (continuous blue curve) registrations: [Left] Trajectory deviations between two different paths (in $\mathrm{mm}$ ); [Right] Absolute intensity differences after registration
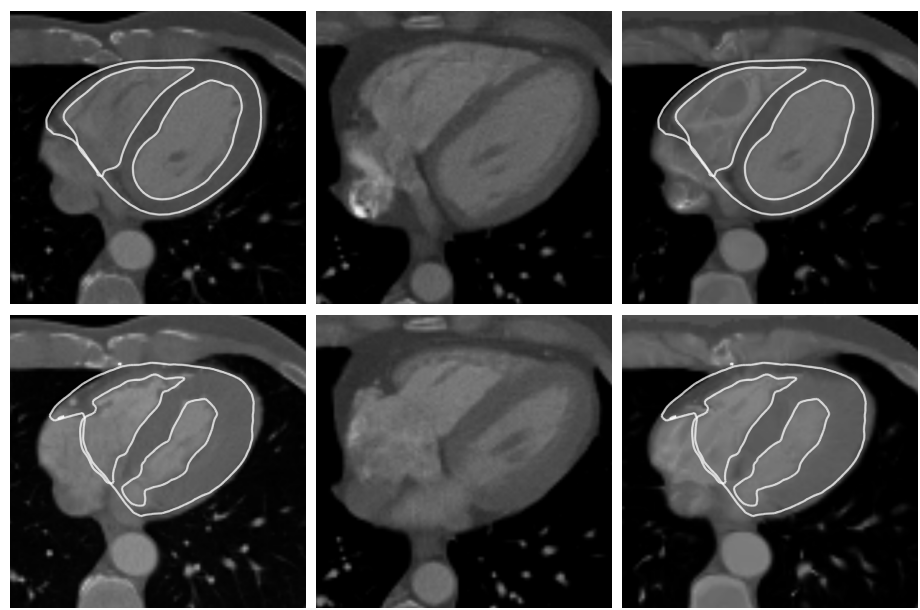

Fig. 3. Results of $4 \mathrm{D}$ registration at ED (Top Row) and ES (Bottom Row): [Left] Reference sequence; [Center] Target sequence before registration; [Right] Target sequence after registration. Segmentations of the endocardium (continuous white lines) are computed in the reference sequence and overlayed over the reference sequence and the transformed target sequence to illustrate the quality of the registration.

$S_{1}^{4 D}$ is computed under trajectory constraints following Equation 3 . Once $S_{1}^{4 D}$ is computed, we can determine the other $S_{j}$ satisfying the trajectory constraints: $S_{j}^{4 D}=M_{1, j}^{\prime} \circ S_{1}^{4 D} \circ M_{1, j}^{-1}$. We compared this $4 \mathrm{D}$ method to the $3 \mathrm{D}$ approach with a direct and independent registration of corresponding frames based on Equation2 and optimized with the Diffeomorphic Demons. On a biprocessor Dual-Core @ $1.86 \mathrm{GHz}$ with $3 \mathrm{~GB}$ RAM, the computation time of direct 3D registration of 20 frames is about 20 minutes. The $4 \mathrm{D}$ registration is about 50 minutes, mainly due to the computation of the tracking (about 20 minutes in each sequence). Both 


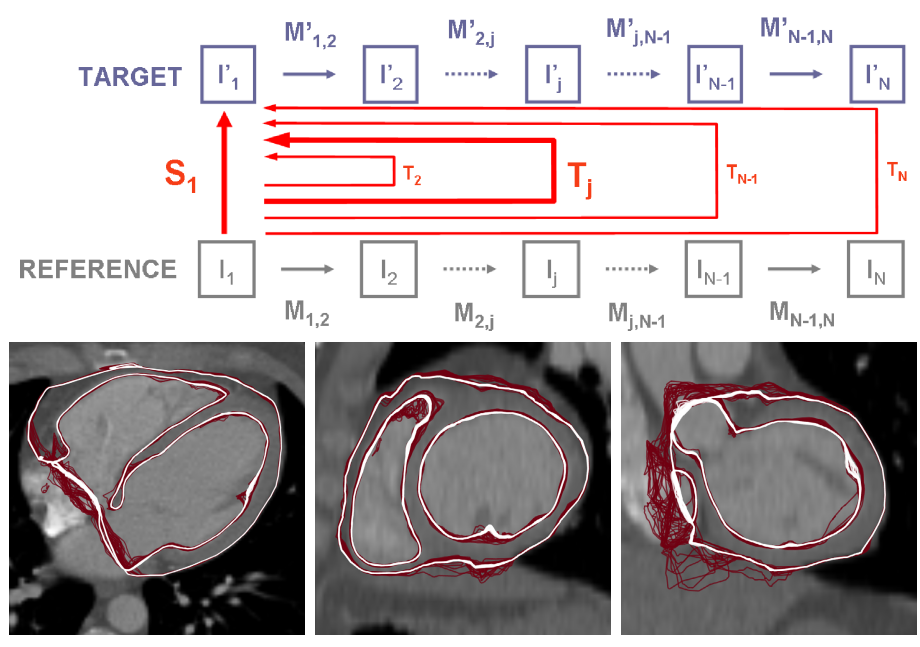

Fig. 4. To visualize the trajectories deviation, segmentations of endocardium and epicardium at the initial time of the reference sequence are propagated to the initial time of the target sequence through different paths of transformations $S_{1}$ and $T_{j}$ for $j>$ 1. [Top Row] The diagram shows the different paths used to propagate the segmentation from $I_{1}$ to $I_{1}^{\prime}$; [Bottom Row] The transformed segmentations using the transformations $S_{j}^{4 D}$ from $4 \mathrm{D}$ registration (continuous white lines) are more consistent than the ones using the transformations $S_{j}^{3 D}$ from $3 \mathrm{D}$ registration (continuous brown lines).

approaches are highly parallelizable. Fig. 2 compares their performances using two metrics: one for the trajectory constraint and one for the image similarity between $I_{j}^{\prime}$ and $I_{j} \circ S_{j}$. For the trajectory constraint, we measure the trajectory deviations $\left\|S_{1}(\nu)-T_{j}(\nu)\right\|$ between two different paths from $I_{1}$ to $I_{1}^{\prime}$ through transformations $S_{1}$ and $T_{i}=M_{1, j}^{\prime-1} \circ S_{j} \circ M_{1, j}$ as shown in Fig. 4. We accumulate it in a histogram for all transformations $T_{j}$. Our method clearly improves the consistency of the transformations over the sequence through the trajectory constraints (mean value $0.20 \mathrm{~mm}$ for $4 \mathrm{D}$ and $2.79 \mathrm{~mm}$ for $3 \mathrm{D}$ ), whereas the error of intensity similarities slightly increases due to the compromise between trajectory and intensity constraints. In Fig. 3. we show the reference, target and transformed target images at two different time points: end of diastole (ED) and end of systole (ES). The segmentations of the endocardium and epicardium in the reference images are overlayed over the reference and transformed target images to visualize the quality of the registration. The segmentations in the transformed target image fit the structures segmented in the reference sequence. We further illustrate in Fig. 4 these results by tracking the segmentation of the endocardium and epicardium at the initial frame of the reference sequence. These segmentations are then transformed to the initial frame of the target sequence using different paths through transformations $T_{1}=S_{1}$ and $T_{j}$ for $j>1$ as shown in the diagram of Fig. 4. The tracking of the segmentations using $4 \mathrm{D}$ registration transformations $S_{j}^{4 D}$ shows a lower dispersion than using $3 \mathrm{D}$ registration 
transformations $S_{j}^{3 D}$. This observation is highly significant in low gradient area where the tracking regularizes the inter-subject registration.

\section{Discussion}

We proposed a framework for the spatio-temporal registration of $4 \mathrm{D}$ sequences (or any time-series images such as longitudinal studies) that non-linearly matches corresponding frames of the sequences and preserves the homology between physical points over time. In this way, the trajectory constraints act as a regularization of shape transformations consistent with motion transformations. The temporal registration strategy still remains to be studied for a complete spatiotemporal alignment. The statistical analysis of the motion and shape transformations across a population would help for a better understanding of inter-subject cardiac motion and shape variabilities.

Acknowledgements. The authors gratefully acknowledge the financial support by Siemens Corporate Research and INRIA for this project.

\section{References}

1. Montagnat, J., Delingette, H.: 4D Deformable Models with Temporal Constraints: Application to 4D Cardiac Image Segmentation. Med. Im. Anal. 9(1) (2005)

2. Shen, D., Sundar, H., Xue, Z., Fan, Y., Litt, H.: Consistent Estimation of Cardiac Motions by 4D Image Registration. In: Duncan, J.S., Gerig, G. (eds.) MICCAI 2005. LNCS, vol. 3750, pp. 902-910. Springer, Heidelberg (2005)

3. Sarrut, D., Delhay, B., Villard, P., Boldea, V., Beuve, M., Clarysse, P.: A Comparison Framework for Breathing Motion Estimation Methods from 4D Imaging. IEEE Trans. Med. Imag. 26(12) (2007)

4. Perperidis, D., Mohiaddin, R., Rueckert, D.: Spatio-Temporal Free-Form Registration of Cardiac MR Image Sequences. Med. Im. Anal. 9 (2005)

5. Singh, M., Basu, A., Mandal, M.: Event Dynamics Based Temporal Registration. IEEE Trans. Med. Im. 9(5) (2007)

6. Caspi, Y., Irani, M.: Spatiotemporal Alignment of Sequences. IEEE Trans. Pat. Anal. Mach. Int. 24(11) (2002)

7. Thirion, J.P.: Image Matching as a Diffusion Process: an Analogy with Maxwell's Demons. Med. Im. Anal. 2(3) (1998)

8. Vercauteren, T., Pennec, X., Perchant, A., Ayache, N.: Non-parametric Diffeomorphic Image Registration with Demons Algorithm. In: Ayache, N., Ourselin, S., Maeder, A. (eds.) MICCAI 2007, Part II. LNCS, vol. 4792, pp. 319-326. Springer, Heidelberg (2007)

9. Guimond, A., Guttmann, C.G., Warfield, S., Westin, C.F.: Deformable Registration of DT-MRI Data Based on Transformation Invariant Tensor Characteristics. In: ISBI (2002)

10. Yeo, B.T.T., Vercauteren, T., Fillard, P., Pennec, X., Golland, P., Ayache, N., Clatz, O.: DTI Registration with Exact Finite-Strain Differential. In: ISBI (2008) 